\title{
Time-reversal-odd phenomena in QCD
}

\author{
P.J. Mulders* \\ Department of Physics and Astronomy, FEW, VU University \\ De Boelelaan 1081, NL-1009 DB Amsterdam, the Netherlands \\ E-mail: mulders@few.vu.nl
}

Single-spin asymmetries were long thought to vanish in high-energy scattering processes because of their specific time-reversal behavior. Time-reversal-odd phenomena, however, appear naturally when one includes effects of intrinsic transverse momenta of partons. The partons, quarks and gluons, enter the description of high-energy scattering processes in correlators which contain quark and gluon fields. The correlators, parameterized in terms of distribution and fragmentation functions, constitute matrix elements of non-local field configurations. For transverse momentum dependent (TMD) correlators the non-locality is along a light-front, in contrast to the non-locality along the light-cone for collinear correlators, integrated over transverse momenta. The TMD correlators require a careful treatment to assure color gauge invariance, leading to nontrivial gauge links connecting the parton fields. These give rise to time-reversal-odd phenomena, showing up as single spin and azimuthal asymmetries. The gauge links, arising from multi-gluon initial and final state interactions, depend on the color flow in the process, which has consequences for issues like universality and factorization.

LIGHT CONE 2008 Relativistic Nuclear and Particle Physics

July 7-11 2008

Mulhouse, France

${ }^{*}$ Speaker. 


\section{Introduction}

The theory of Quantum Chromodynamics (QCD), underlying the quark and gluon structure of hadrons, is invariant under time-reversal (T), which allows the distinction of T-even and Todd quantities. Single-spin asymmetries are specific examples of T-odd observables. In general, however, $\mathrm{T}$ cannot be used as a constraint for the (complex) S-matrix. At high energies the cross sections of a (hard) scattering process factorizes in (hadronic) soft parts, which we will discuss in detail, and a partonic cross section. The partonic cross sections at tree-level are T-even with T-odd phenomena coming in at order $\alpha_{s}$ beyond tree-level. Thus, one expects at leading order T-even soft parts (or an even number of T-odd parts) to be relevant in double spin asymmetries and T-odd soft parts (at least an odd number of them) to be relevant in single spin asymmetries.

In situations in which one integrates over all transverse momenta one has collinear correlators describing the soft parts, parametrized in terms of distribution functions (DF) and fragmentation functions (FF) that depend only on a collinear momentum fraction. For spin 0 and spin $1 / 2$ hadrons these collinear correlators are only T-even. This leads to the expectation that T-odd phenomena are absent in high-energy scattering processes. This indeed is true for processes with only one hadron in the initial state. The example of this is of course inclusive deep inelastic scattering, $e p \rightarrow e X$. For hadron-hadron collisions or semi-inclusive fragmentation processes even a factorized description allows T-odd phenomena.

Single spin asymmetries (SSA) are known to exist, persisting at high energies [1]. There are many studies of mechanisms that lead to single spin asymmetries (SSA) in hard scattering processes, with Sivers [2] and Collins effects [3,4] serving as notable examples in the supposedly simple case of leptoproduction. As alluded to, in collinear approximation (integrating over all transverse momenta) all leading twist distribution (and fragmentation) functions only depend on the longitudinal momentum fraction $x$ (or $z$ ) are T-even. For spin 0 and spin $1 / 2$ hadrons this implies e.g. that polarized quarks are only found in polarized hadrons (and vice versa). Single spin asymmetries (SSA) can occur but restricting ourselves to collinear correlators they require twist-three correlators involving quark-gluon matrix elements [5]. For higher spins, e.g. for spin 1 hadrons, one also has T-odd collinear twist-two fragmentation functions [6]. Returning to the (T-odd) quark-gluon matrix elements, one finds that they can appear at leading order in the specific limit of a zero-momentum gluon, referred to as gluonic pole matrix elements such as the QiuSterman matrix elements [7]. Also in model calculations the effects of these soft gluon interactions between the target remnant and the hard part have been demonstrated, giving rise to distinct effects for initial or final state interactions [8].

Going beyond the collinear approximation, the incorporation of intrinsic transverse momenta of partons provides another mechanism to generate leading order SSA, which can be traced back to correlations between the intrinsic transverse motion and spin of partons and/or hadron [2, 3]. The effects are described by transverse momentum dependent (TMD) distribution functions $[9,10,11]$, containing both T-even and T-odd parts and depending on longitudinal momentum fraction $x$ and the transverse momentum $p_{T}$ as appearing in the Sudakov decomposition $p=x P+p_{T}$ (or $p=$ $(1 / z) P+p_{T}$ for fragmentation). The TMD correlators include Wilson lines, which besides ensuring gauge-invariance are in the case of distribution functions the sole cause of T-odd contributions. Upon $p_{T}$-integration one finds after weighing with $p_{T}$ the socalled transverse moments of the TMD 


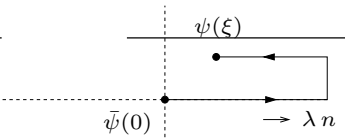

(a) $\Phi^{[+]}$

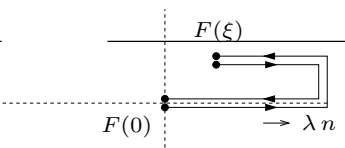

(c) $\Gamma^{[+,+]}$

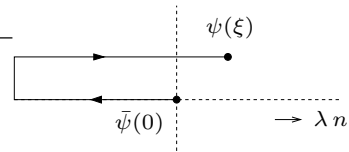

(b) $\Phi^{[-]}$
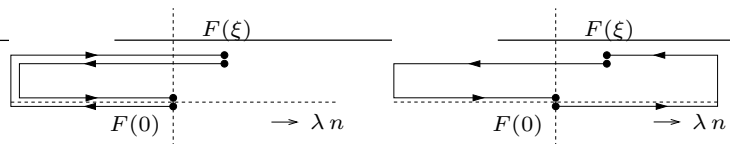

(e) $\Gamma^{[+,-]}$

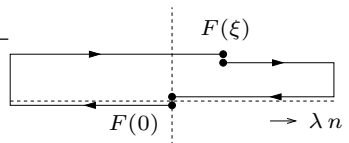

(f) $\Gamma^{[-,+]}$

Figure 1: Simplest structures (without loops) for gauge links and operators in quark correlators (a)-(b) and gluon correlators (c)-(f).

distribution functions, which can be separated into T-even and T-odd parts that are universal and of which the T-odd part can be identified with the gluonic pole matrix elements.

\section{Transverse momentum dependent (TMD) correlators}

The TMD distribution functions are projections of the TMD quark correlator defined on the light-front (LF: $\xi \cdot n \equiv 0)$

$$
\left.\Phi_{i j}^{[C]}\left(x, p_{T} ; n\right)=\int \frac{d(\xi \cdot P) d^{2} \xi_{T}}{(2 \pi)^{3}} e^{i p \cdot \xi}\left\langle P, S\left|\bar{\psi}_{j}(0) \mathscr{U}_{[0 ; \xi]}^{[C]} \psi_{i}(\xi)\right| P, S\right\rangle\right\rfloor_{\mathrm{LF}} .
$$

The Wilson line or gauge link $\mathscr{U}_{[\eta ; \xi]}^{[C]}=\mathscr{P} \exp \left[-i g \int_{C} d s \cdot A^{a}(s) t^{a}\right]$ is a path-ordered exponential along the integration path $C$ with endpoints at $\eta$ and $\xi$, ensuring gauge-invariance. In the TMD correlator 2.1 the integration path $C$ in the gauge link turns out to be process-dependent.

In a diagrammatic approach the Wilson lines arise by resumming all collinear gluons exchanged between the soft and the hard partonic parts of the hadronic process. The integration path $C$ is fixed by the (color-flow structure of) the hard partonic scattering [12]. Basic examples (see Fig. 1) are semi-inclusive deep-inelastic scattering (SIDIS) where for the quark correlator the resummation of all final-state interactions leads to the future pointing Wilson line $\mathscr{U}^{[+]}$, and DrellYan scattering where the initial-state interactions lead to the past pointing Wilson line $\mathscr{U}^{[-]}$. These links connect the parton fields in the correlator, running along the light-like direction $n$, conjugate to $P$ (satisfying $P \cdot n=1$ and $n^{2}=0$ ) and closing in the transverse direction at lightcone infinity [13]. For gluons the correlators including links are given by [14]

$$
\Gamma_{\alpha \beta}^{\left[C, C^{\prime}\right]}\left(x, p_{T} ; n\right)=\left.\int \frac{d(\xi \cdot P) d^{2} \xi_{T}}{(2 \pi)^{3}} e^{i p \cdot \xi}\left\langle P, S\left|\operatorname{Tr}\left(F_{\beta}^{n}(0) U_{[0, \xi]}^{[n, C]} F_{\alpha}^{n}(\xi) U_{[\xi, 0]}^{\left[n, C^{\prime}\right]}\right)\right| P, S\right\rangle\right|_{L F},
$$

with the simplest possibilities also shown in Fig. 1.

\section{Observables}

Considering intrinsic transverse momenta makes sense because it is possible to access the effects of them in experiments. The collinear fractions $(x$ or $z$ ) in the Sudakov expansion of the 
parton momenta can be related to kinematical ratios of hard momenta (e.g. $x \approx x_{B}=Q^{2} / 2 P \cdot q$ and $z \approx z_{h}=P_{h} \cdot P / P \cdot q$ in semi-inclusive deep inelastic scattering) up to $O\left(1 / Q^{2}\right)$ corrections. Therefore the quantity $q_{T}=q+x_{B} P-P_{h} / z_{h} \approx k_{T}-p_{T}$ can be measured in semi-inclusive deep inelastic scattering (SIDIS), $\gamma^{*}(q)+N(P) \rightarrow h\left(P_{h}\right)+X$. It is zero at leading order $(O(Q)$ in the hard scale), but relates to the intrinsic transverse momenta at $O(M)$. Essential in establishing the relation to the intrinsic transverse momenta is the assumption of a particular partonic subprocess, in the case of SIDIS $\gamma^{*}(q)+q(p) \rightarrow q(k)$. The vector $q_{T}$ is the transverse momentum of $q$ in a frame in which $P$ and $P_{h}$ are chosen parallel or (experimentally more useful) related to the transverse momentum of $P_{h}, q_{T}=-P_{h \perp} / z_{h}$ in a frame in which $q$ and $P$ are chosen parallel. With $Q_{T}^{2}=-q_{T}^{2}$, one needs TMD functions when $Q_{T} \sim O(M)$ and one needs a collinear description involving a subprocess with one more parton radiated off when $Q_{T} \sim O(Q)$. Matching of these approaches was condidered in Ref. [15]. Not only in electroweak processes like SIDIS or the Drell-Yan process can transverse momenta be accessed, but also one can consider inclusive hadron-hadron scattering. The experimental signature in this case is the non-collinearity of the produced particles/jets in the plane perpendicular to the colliding beam particles, outlined in detail in Ref. [16].

Accessing intrinsic transverse momenta in most cases requires a study of azimuthal dependence in high energy processes. Although the effects are in principle not suppressed by powers of the hard scale in comparison with the leading collinear treatment, it requires measuring hadronic scale quantities (transverse momenta) in a high momentum environment. We already mentioned that for the explanation of single spin asymmetries time reversal invariance plays an important role: The T-invariance of QCD allows to distinguish quantities and observables according to their T-behavior. Leading twist collinear correlators $\Phi(x)$ and $\Gamma(x)$ (i.e. leading in an expansion in the inverse hard scale) are all T-even. For the TMD correlators, however, the T-operation interchanges $\Phi^{[+]}\left(x, p_{T}\right) \leftrightarrow \Phi^{[-]}\left(x, p_{T}\right)$ (with similar relations holding for gluon TMD correlators), allowing us to construct correlators with T-even and T-odd operator combinations and providing an explanation of SSA.

We note that for fragmentation functions the appearance of an hadronic out-state in the definition, prohibits the use of T-symmetry as a constraint in the first place. One thus in principle always has both T-even and T-odd functions appearing in the parametrization, although these do only appear at subleading twist or for TMD correlators. It is for many purposes still useful to separate the correlators into two classes containing T-even or T-odd operator combinations in analogy with the case of distributions, referred to as naive T-even or naive T-odd. But one must be aware that both the naive T-even and naive T-odd correlators contain T-even and T-odd parts and corresponding FFs in the parametrization.

\section{TMD treatment}

As already referred to in section 2 the gauge links in the correlators are the result of resumming leading matrix elements with collinear gluons. The presence of links, differing for each partonic sub-diagram and its color-flow, results in the following generic expression for a hard cross section at measured $q_{T}$ (involving in general complex diagram-dependent gauge-link paths),

$$
\frac{d \sigma}{d^{2} q_{T}} \sim \sum_{D, a b c \ldots} \Phi_{a}^{\left[C_{1}(D)\right]}\left(x_{1}, p_{1 T}\right) \Phi_{b}^{\left[C_{2}(D)\right]}\left(x_{2}, p_{2 T}\right) \hat{\sigma}_{a b \rightarrow c \ldots}^{[D]} \Delta_{c}^{\left[C_{1}^{\prime}(D)\right]}\left(z_{1}, k_{1 T}\right) \ldots+\ldots
$$


where the sum $D$ runs over diagrams distinguishing also the color flow and $a b c \ldots$ is the summation over quark and antiquark flavors and gluons. Dirac and Lorentz indices, as well as traces are suppressed. The ellipsis at the end indicate contributions of other hard processes.

The results for cross sections after integration over the transverse momenta $q_{T}$ also allows integration over the partonic transverse momenta and one obtains the path-independent collinear correlators $\Phi(x)$ rather than the path-dependent TMD correlators $\Phi^{[C(D)]}\left(x, p_{T}\right)$. Thus, from Eq. 4.1 one gets the well-known collinear result

$$
\sigma \sim \sum_{a b c \ldots} \Phi_{a}\left(x_{1}\right) \Phi_{b}\left(x_{2}\right) \hat{\sigma}_{a b \rightarrow c \ldots} \Delta_{c}\left(z_{1}\right) \ldots+\ldots
$$

where $\hat{\sigma}_{a b \rightarrow c \ldots}=\sum_{D} \hat{\sigma}_{a b \rightarrow c \ldots}^{[D]}$ is the partonic cross section.

Constructing a weighted cross section (azimuthal asymmetry) by including a weight $q_{T}^{\alpha}$ in the $q_{T}$-integration leads to

$$
\left\langle q_{T}^{\alpha} \frac{d \sigma}{d^{2} q_{T}}\right\rangle \sim \sum_{D, a b c \ldots} \Phi_{\partial a}^{\alpha\left[C_{1}(D)\right]}\left(x_{1}\right) \Phi_{b}\left(x_{2}\right) \hat{\sigma}_{a b \rightarrow c \ldots}^{[D]} \Delta_{c}\left(z_{1}\right) \ldots+\ldots
$$

containing a number of terms in each of which for one of the partons a transverse moment appears,

$$
\Phi_{\partial}^{\alpha[C]}(x)=\int d^{2} p_{T} p_{T}^{\alpha} \Phi^{[C]}\left(x, p_{T}\right)=\widetilde{\Phi}_{\partial}^{\alpha}(x)+C_{G}^{[U(C)]} \pi \Phi_{G}^{\alpha}(x, x) .
$$

These transverse moments still contain a path-dependence, so Eq. 4.3 cannot be simplified immediately but, as also shown in the above equation, the path dependence is contained in a (gluonic pole) factor $C_{G}$, which can easily be calculated. The first term, $\widetilde{\Phi}_{\partial}(x)$, is a collinear correlator containing matrix elements with T-even operators, while $\Phi_{G}\left(x, x-x_{1}\right)$ is a collinear correlator with a structure of a quark-gluon-quark correlator involving the gluon field $F^{n \alpha}$. In Eq. 4.4 one needs the zero-momentum $\left(x_{1}=0\right)$ limit for the gluon momentum. This matrix element is known as the gluonic pole matrix element. The operators involved are T-odd. Both collinear correlators on the RHS in Eq. 4.4 are link-independent. Using this decomposition one can write down a parton-model like expansion for the single-weighted cross section $\left\langle q_{T}^{\alpha} \sigma\right\rangle$ in Eq. 4.3 in which $\widetilde{\Phi}_{\partial}^{\alpha}(x)$ is multiplied with the partonic cross section, while $\pi \Phi_{G}^{\alpha}(x, x)$ is multiplied with the gluonic pole cross section,

$$
\hat{\sigma}_{[a] b \rightarrow c \ldots}=\sum_{D} C_{G}^{[U(C(D))]} \hat{\sigma}_{a b \rightarrow c \ldots}^{[D]},
$$

which besides the normal partonic cross sections constitutes a different gauge-invariant combination of the squared amplitudes [17]. For a number of processes, the consequences for (weighted) azimuthal single spin asymmetries have been investigated [18]. For more complex weightings or trying to stay at the unintegrated level, one has to make additional assumptions outlined in Ref. [14]. In this paper also the split-up of TMD functions in

$$
\Phi^{[U]}\left(x, p_{T}\right)=\Phi^{[\mathrm{even}]}\left(x, p_{T}\right)+G_{G}^{[U]} \Phi^{[\mathrm{odd}]}\left(x, p_{T}\right)+\delta \Phi^{[U]}\left(x, p_{T}\right),
$$

with $\Phi^{[\text {even/odd }]}=\frac{1}{2}\left(\Phi^{[+]} \pm \Phi^{[-]}\right)$is discussed. The even and odd combinations are constructed from the correlators with simple links shown in Fig. 1. Upon integration one has $\Phi^{[\text {even }]}(x)=\Phi(x)$, 
$\Phi^{[\text {odd }]}(x)=0, \Phi_{\partial}^{\alpha[\text { even }]}(x)=\widetilde{\Phi}_{\partial}^{\alpha}(x)$, and $\Phi_{\partial}^{\alpha \text { [odd }]}(x)=\pi \Phi_{G}^{\alpha}(x, x)$. The additional terms is referred to as junk TMD satisfying $\delta \Phi^{[U]}(x)=\delta \Phi_{\partial}^{\alpha[U]}(x)=0$.

Similar combinations of even and odd correlators can be constructed from the simplest gluon correlators [14], although one must be aware that there are two types of gluonic pole matrix elements [19] corresponding to the two different ways to construct color singlets for three gluons and correspondingly, there are for instance two distinct gluon-Sivers distribution functions.

\section{Conclusions}

The approach to understand T-odd observables like single spin asymmetries via the TMD correlators and the non-trivial gauge link structure unifies a number of approaches to understand such observables, in particular the collinear approach and the inclusion of soft gluon interactions. Although the treatment of fragmentation correlators also separates into naive T-even and naive T-odd parts with T-even and T-odd operator structure respectively, the gluonic pole contributions (naive T-odd parts) in the case of fragmentation might very well vanish. Indications come from the soft-gluon approach [20] and a recent spectral analysis in a spectator model approach [21].

\section{References}

[1] D. L. Adams et al, Comparison of spin asymmetries and cross-sections in $\pi^{0}$ production by $200 \mathrm{Gev}$ polarized anti-protons and protons. Phys. Lett., B261:201-206, 1991; D. L. Adams et al, Analyzing power in inclusive $\pi^{+}$and $\pi^{-}$production at high $x_{F}$ with a $200 \mathrm{Gev}$ polarized proton beam. Phys. Lett., B264:462-466, 1991; A. Bravar et al, Single-spin asymmetries in inclusive charged pion production by transversely polarized antiprotons. Phys. Rev. Lett., 77:2626-2629, 1996; A. Airapetian et al, Single-spin azimuthal asymmetries in electroproduction of neutral pions in semi-inclusive deep-inelastic scattering. Phys. Rev., D64:097101, 2001; S. S. Adler et al, Mid-rapidity neutral pion production in proton proton collisions at $\sqrt{s}=200$ Gev. Phys. Rev. Lett., 91:241803, 2003; J. Adams et al, Cross sections and transverse single-spin asymmetries in forward neutral pion production from proton collisions at $\sqrt{s}=200 \mathrm{Gev}$. Phys. Rev. Lett., 92:171801, 2004; A. Airapetian et al, Single-spin asymmetries in semi-inclusive deep-inelastic scattering on a transversely polarized hydrogen target. Phys. Rev. Lett., 94:012002, 2005.

[2] Dennis W. Sivers, Single spin production asymmetries from the hard scattering of point-like constituents. Phys. Rev., D41:83, 1990; Dennis W. Sivers, Hard scattering scaling laws for single spin production asymmetries. Phys. Rev., D43:261-263, 1991.

[3] John C. Collins, Fragmentation of transversely polarized quarks probed in transverse momentum distributions. Nucl. Phys., B396:161-182, 1993.

[4] John C. Collins, Steve F. Heppelmann, and Glenn A. Ladinsky, Measuring transversity densities in singly polarized hadron hadron and lepton - hadron collisions. Nucl. Phys., B420:565-582, 1994.

[5] R. L. Jaffe and Xiang-Dong Ji, Novel quark fragmentation functions and the nucleon's transversity distribution. Phys. Rev. Lett., 71:2547-2550, 1993.

[6] A. Bacchetta and P. J. Mulders, Deep inelastic leptoproduction of spin-one hadrons. Phys. Rev., D62:114004, 2000.

[7] Jian-wei Qiu and George Sterman, Single transverse spin asymmetries. Phys. Rev. Lett., 67:2264-2267, 1991. 
[8] Stanley J. Brodsky, Dae Sung Hwang, and Ivan Schmidt, Final-state interactions and single-spin asymmetries in semi-inclusive deep inelastic scattering. Phys. Lett., B530:99-107, 2002.

[9] P. J. Mulders and R. D. Tangerman, The complete tree-level result up to order 1/Q for polarized deep-inelastic leptoproduction. Nucl. Phys., B461:197-237, 1996.

[10] Daniel Boer and P. J. Mulders, Time-reversal odd distribution functions in leptoproduction. Phys. Rev., D57:5780-5786, 1998.

[11] Alessandro Bacchetta et al, Semi-inclusive deep inelastic scattering at small transverse momentum. JHEP, 02:093, 2007.

[12] C. J. Bomhof, P. J. Mulders, and F. Pijlman, The construction of gauge-links in arbitrary hard processes. Eur. Phys. J., C47:147-162, 2006.

[13] Andrei V. Belitsky, X. Ji, and F. Yuan, Final state interactions and gauge invariant parton distributions. Nucl. Phys., B656:165-198, 2003.

[14] C. J. Bomhof and P. J. Mulders, Non-universality of transverse momentum dependent parton distribution functions. Nucl. Phys., B795:409-427, 2008.

[15] Alessandro Bacchetta, Daniel Boer, Markus Diehl, and Piet J. Mulders, Matches and mismatches in the descriptions of semi-inclusive processes at low and high transverse momentum. JHEP, 08:023, 2008.

[16] Daniel Boer and Werner Vogelsang, Asymmetric jet correlations in p p(pol.) scattering. Phys. Rev., D69:094025, 2004.

[17] C. J. Bomhof and P. J. Mulders, Gluonic pole cross sections and single spin asymmetries in hadron hadron scattering. JHEP, 02:029, 2007.

[18] Alessandro Bacchetta, Cedran Bomhof, Umberto D’Alesio, P. J. Mulders, and F. Murgia, The Sivers single-spin asymmetry in photon - jet production. Phys. Rev. Lett., 99:212002, 2007; D. Boer, P. J. Mulders, and C. Pisano, T-odd effects in photon-jet production at the Tevatron. Phys. Lett., B660:360-368, 2008; D. Boer, C. J. Bomhof, D. S. Hwang, and P. J. Mulders, Spin asymmetries in jet-hyperon production at LHC. Phys. Lett., B659:127-136, 2008; C. J. Bomhof, P. J. Mulders, W. Vogelsang, and F. Yuan, Single-transverse spin asymmetry in dijet correlations at hadron colliders. Phys. Rev., D75:074019, 2007; Zhun Lu and Ivan Schmidt, Azimuthal angle dependence of di-jet production in unpolarized hadron scattering. 2008.

[19] Xiang-Dong Ji, Gluon correlations in the transversely polarized nucleon. Phys. Lett., B289:137-142, 1992.

[20] John C. Collins and Andreas Metz, Universality of soft and collinear factors in hard- scattering factorization. Phys. Rev. Lett., 93:252001, 2004.

[21] L. P. Gamberg, A. Mukherjee, and P. J. Mulders, Spectral analysis of gluonic pole matrix elements for fragmentation. Phys. Rev., D77:114026, 2008. 